\title{
Modeling the Interactions between the BDNF Peptides and TrkB Receptor
}

\author{
Dharmaiah Devarapalli \\ Associate Professor \\ Department of computer \\ Science and Engineering \\ Vignan's Institute of Information \\ Technology (VIIT) \\ Visakhapatnam \\ Andhra Pradesh, India -530 \\ 049
}

\author{
Allam Appa Rao \\ Director \\ CR Rao Advanced Institute for \\ Mathematics, Statistics \& \\ Computer Science (AIMSCS) \\ University of Hyderabad \\ Campus \\ Hyderabad \\ Andhra Pradesh, \\ India - 500046
}

\author{
G R Sridhar \\ DM, FACE, FRCP \\ Endocrine \& Diabetes Centre \\ 15-12-15 \\ Krishna Sagar \\ Visakhapatnam \\ Andhra Pradesh \\ India-530002
}

\begin{abstract}
The BDNF/TrkB signaling system which plays a major role in the regulation of neuronal activity. This neuronal regulation influences the potential role of this system in the therapeutic efficacy of many neurological and psychiatric disorders. Despite these roles decreased levels of BDNF are associated with diabetes and obesity which can be regulated by increasing the insulin sensitivity and glucose tolerance. BDNF mediated signaling through phosphoinositide 3-kinase (PI3K) pathway plays a key role in insulin sensitivity. These beneficial effects of BDNF as antidiabetic agents can be enhanced by the activating BDNF\TrkB signaling. These multiple functionality of BDNFITrkB complex relies on the protein-protein interactions where those interactions are important in designing pharmacological targets. This apporach focus the use of BDNF peptides in place of BDNF protein in binding to the TrkB receptor. Protein-peptide docking studies were performed to know the interactions of the TrkB and BDNF peptides. Docking studies were done using ZDOCK pro (Accerlys Discovery studio). The interacting amino acids residues identified at the binding site were THR 306, LYS 308, CYS 5 ASP 370.
\end{abstract}

\section{General Terms}

Docking Studies, Receptor, Peptides,

\section{Keywords}

BDNF\TrkB signaling system, Protein-protein interactions, Protein-peptide interaction, Diabetes mellitus.

\section{INTRODUCTION}

The biomolecular interactions in many regulatory processes are often a consequence of specific protein-protein contacts, exerting their central role in controlling many cellular events[1-3]. The fundamental processes governed by these protein-protein interactions are signal transduction, cell cycle proliferation, cellular architecture biosynthetic and degradation pathways and

their regulations, immune response etc[4]. The cellular and molecular level understandings of these events can be known by the quantitative structural and functional analysis of the interactions of the protein complexes[5-6]. The findings of how the interaction of protein complexes enable these biological processes help researchers to identify the underlying causes of many diseases and ultimately develop targeted therapeutic strategies. The ubiquitous pathophysiological role of PPIs in a large number of diseases, make them attractive targets for pharmaceutical intervention[7-9]. The quantitative characterization of the interactions include locating the binding sites on the surfaces of both proteins, detecting the residues that mediate the interaction and their differential thermodynamic contribution to the interaction, measuring the affinity of the interaction and studying the biological role of the interaction in cells. A major rate-limiting step in PPI is studying the conformational changes between two full-length proteins which is not so easier [10]. PPIs having flat surface and featureless topologies, exhibiting great conformational dynamics and surface adaptively to accommodate one or more protein partners, whose interactions distribute over large surface areas[11]. In order to overcome this here we presented the use of protein derived peptides in place of one of the full length protein. The main shortcoming of using linear peptides for therapeutic purposes is their wide conformational range and short lifetime. Studying the interaction between a full-length protein and a short peptide derived from the protein's partner is technically easier than studying the interaction between two full-length proteins. The binding of a peptide to a full-length protein may also be used to characterize conformational changes that occur on one partner participating in a PPI. Protein-peptide interactions are also studied for biophysical characterization of PPIs, such as quantifying the affinity, kinetics and thermodynamics of the interaction [10].

BDNF is a member of the Neurotrophic factor family, expressed in the nervous system and periphery which plays a key role in regulating and maintenance of neuronal outgrowth, differentiation, synaptic connection, and neuronal repair [12]. In addition to its critical role in helping shape the nervous system during development, BDNF is of particular therapeutic interest because of its Neurotrophic actions been implicated in the pathogenesis of neurodegenerative and psychiatric disorders [13]. Responses to BDNF are mediated by a tyrosine receptor kinaseB, TrkB. Binding of BDNF rapidly activate TrkB activity, leading to receptor dimerization and activation of the catalytic tyrosine protein kinase domains. The dimerized Trk receptors autophosphorylate several key intracellular tyrosine residues, which rapidly initiates intracellular signaling cascades such as mitogen-activated protein kinase, phosphatidylinositol 3-kinase, and phospholipase C-[14]. Beyond the importance of BDNF in neurological development and synaptic plasticity and its role in learning and memory[15], there is evidence that demonstrates that it is also essential for body weight control and energy homeostasis. Low levels of BDNF have been found in individuals with obesity and type 2 diabetes[16] . BDNF treatment to obese and diabetic conditions significantly 
suppressed the blood glucose, food consumption, and dietary body weight gain, while also enhancing the energy expenditure, glucose and lipid metabolism etc.[17-19] BDNF exhibits hypophagic and hypoglycemic effects in obese hyperglycemic conditions indicating its antiobesity and antidiabetic effects $[16,20]$. However, in humans with type 2 diabetes, levels of BDNF were decreased independent of obesity, suggesting that BDNF may regulate obesity and insulin resistance via different mechanisms, particularly through the insulin-stimulated phosphoinositide 3kinase(PI3K) pathway important not only to neuronal survival and synaptic plasticity but also to insulin receptor signalling and insulin resistance. Here the coupling of BDNF\TrkB signaling system to PI3K pathway increases insulin sensitivity by preventing pancreatic exhaustion by maintaining the histological cellular organization of $\beta$-cells and non- $\beta$-cells in pancreatic islets and by restoring the level of insulin-secreting granules in $\beta$-cells. These findings of BDNF in regulating insulin sensitivity makes them as important therapeutic targets for treating diabetes and may provide better antidiabetic agents.

\section{MATERIAL AND METHODOLOGY 2.1. Preparing protein and BDNF peptides}

The initial crystal structure of TRKB with PDB entry 1WWB containing 103 amino acid residues at a resolution of 2.10 was taken from the PDB database and energy minimization was carried out using Discovery studio. The BDNF peptides were constructed using fragment builder in Discovery Studio. The peptides taken were I-(R)-I-D-T-S- $X$-V- $X$-T-L-T-I-K-R-GR(peptide 1), Y-E-T-K-X-N-P-M-G-Y-T-K-E-G-XR-G-I-D$\mathrm{K}$ ((peptide 2), and S-V-X-D-S-I-S-E(peptide 3).the constructed peptides were carried out with energy minimization by applying steepest descent method followed by conjugant gradient method until the convergence gradient was satisfied. The prepared protein and peptides were further processes for docking studies.

\subsection{Protein-Peptide Docking}

For protein-peptide docking studies, we employed the dock proteins protocol (Discovery Studio) to generate a set of possible configurations for the BDNF peptides and TrkB complex. The ZDOCK protocol is used for docking the BDNF peptides to TrkB and the RDOCK protocol for the subsequent refinement of docked poses. Zdock is a rigid-body docking algorithm using a Fast Fourier Transform (FFT) to perform an exhaustive six-dimensional search in the translational and rotational space between the two molecules (Chen and Weng, 2002; Chen andWeng, 2003). Each protein is projected into a three-dimensional grid and different values are assigned to the cells of the grid, representing the surface or the interior of the molecules. The rotational search sampling grid can use a 15 degree grid which samples a total of 3600 docked poses, or a 6 degree grid which samples a total of 54,000 poses for more accurate results. Zdock searches orientational space by rotating the ligand around its geometric center with the receptor protein kept fixed in space. For each sampled angle only the ligand translation corresponding to the best geometric match between the two proteins is retained. As part of the ZDOCK protocol, the ZRANK function is used to rerank the docked poses. The obtained configurations for the complex are ranked based on a scoring function combining shape complementarity, desolvation energy, and electrostatics. The RDOCK protocol can be used subsequently for further refinement of the docked poses, using a CHARMm-based energy minimization scheme for the optimization of intermolecular interactions with a scoring function being composed of a CHARMm electrostatic energy term and a desolvation energy term. For both complexes, the best pose of the docking prediction reproduced the X-ray structure within RMSD of $1 \mathrm{~A}$.

In the initial stage of ZDOCK, peptides of BDNF and the receptor $\mathrm{TrkB}$ are treated as rigid bodies and all six rotational and translational degrees of freedom are fully explored with scoring functions that are tolerant to conformational changes. No contact information was used, which can filter hits or block residues during the search. An evenly distributed Euler angular step of $15 \mathrm{deg}$ was used for rotational search, which results in 3600 poses. In the RDOCK refinement stage, the 2000 best poses of near native structures obtained in the initial stage were refined and re-ranked using a more detailed energy function that takes into account conformational changes as well as a salvation term.

\section{RESULTS AND DISCUSSION}

Protein-peptide docking studies were done for the prediction of numerous possible BDNF peptide /TrkB complex structures of which only a few of them resembling the native structure. Choosing the near-native structures from the generated set is a key element in predicting correct binding mode of the protein complexes. The PDB structure of TrkB, 1WWB and the BDNF peptides were taken for docking studies. In the initial stage the rigid body algorithm ZDOCK was used to generate protein-protein complex structures. This docking approach generated a total of 3600 protein poses (hits) for the entire study as ZDOCK outputs 2000 protein poses per job. Once ZDOCK has made its predictions, the 2000 protein poses must be refiltered for further processing. In the second approach a more flexible energy minimization algorithm RDOCK was used for complex structure re-ranking and refinement stage. Based on these ranking the best protein pose was selected for analyzing the binding mode of the BDNF-TRKB complex and interacting amino acid residues.

Figure 1 illustrates the top ranking pose of predicted BDNF peptide - TRKB complex conformations that reveal the correct binding mode of the protein. After the RDOCK refinement process, among the binding interfaces of the protein the side chain movements re observed and with unchanged conformations in the backbone conformation. Several intermolecular hydrogen bonds consistently form between the active site regions of the BDNF and TRKB complexes. 


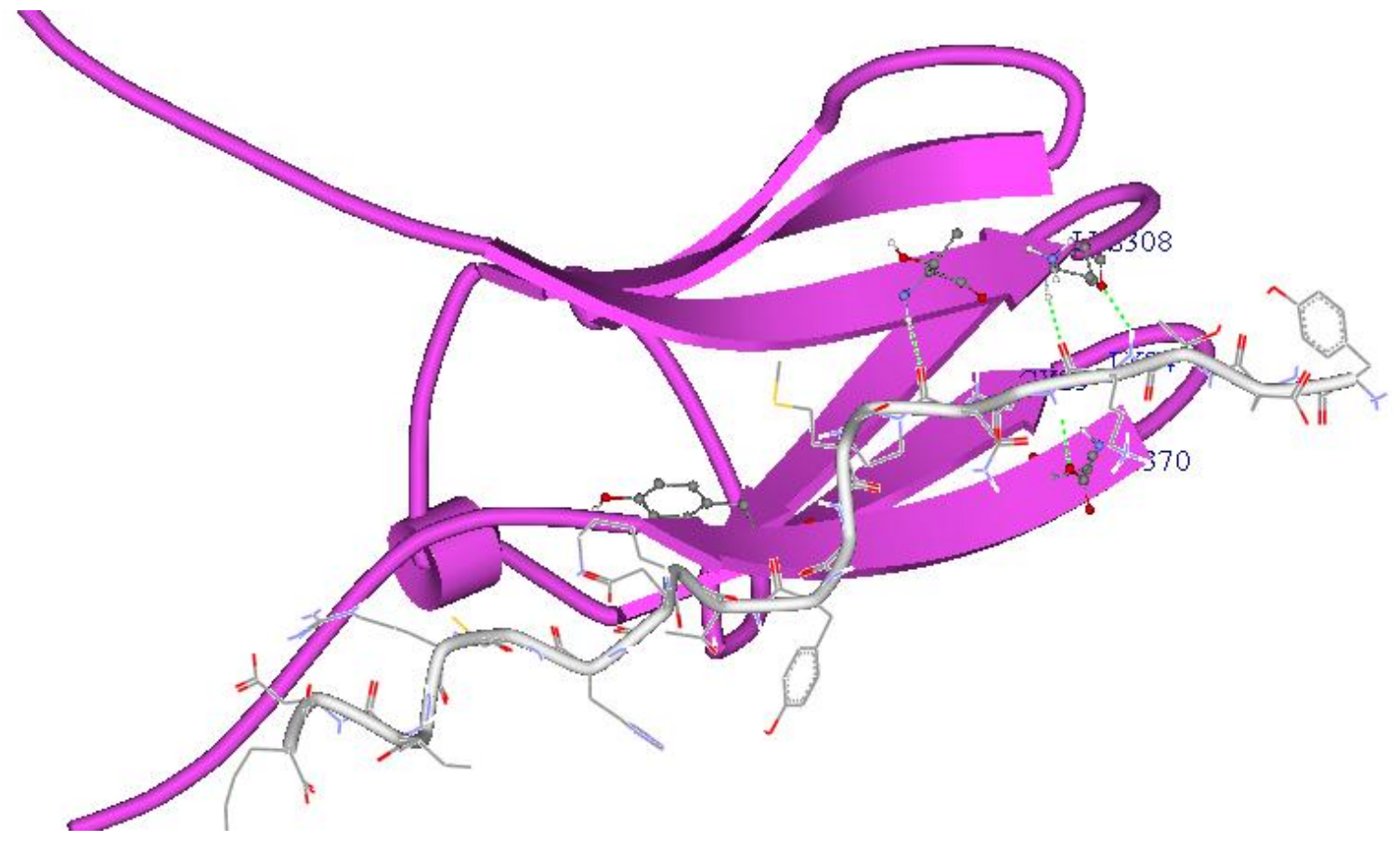

Figure 1. Top rank BDNF-TRKB complex, ZDOCK pose

Remarkably, for the BDNFpeptide-TRKB complex, the first ranked pose out of the 50 RDOCK predicted poses are clustered around the common binding mode. This finding for a BDNFpeptide-TRKB complexes is consistent with computational free energy calculations conducted for knowing the interacting residues of the BDNF peptide3-TRKB complex, where it was found that interactions amino acids were THR 306, ASN 6, LYS 308, LYS 4, CYS 5, ASP 370 which are the major contributors to the overall stability of the complexes.

Table 1. Bdnf-Petides interaction energy calculations with TrkB Receptor.

\begin{tabular}{|c|c|c|c|c|c|c|c|c|}
\hline $\begin{array}{c}\text { Peptide } \\
\text { s }\end{array}$ & $\begin{array}{c}\text { Rec } \\
\text { epto } \\
\mathbf{r}\end{array}$ & $\begin{array}{c}\text { Pose } \\
\mathbf{N u} \\
\mathbf{m}\end{array}$ & $\begin{array}{c}\text { ZDo } \\
\mathbf{c k} \\
\mathbf{s c o r} \\
\mathbf{e}\end{array}$ & $\begin{array}{c}\text { ZRank } \\
\text { Score }\end{array}$ & E_vdw1 & $\begin{array}{c}\text { E_Vdw } \\
\mathbf{2}\end{array}$ & $\begin{array}{c}\text { E_ele } \\
\mathbf{c 2}\end{array}$ & E-sol \\
\hline $\begin{array}{c}\text { IRIDTS } \\
\text { CVCTL } \\
\text { TIKRG } \\
\text { R }\end{array}$ & $\begin{array}{c}\text { TrK } \\
\text { B }\end{array}$ & 219 & 9.1 & -31.69 & -52.055 & -53.802 & -2.891 & -10.5 \\
\hline $\begin{array}{c}\text { YETKC } \\
\text { NPMG } \\
\text { YTKEH } \\
\text { CRGID } \\
\text { K }\end{array}$ & $\begin{array}{c}\text { TrK } \\
\text { B }\end{array}$ & 170 & 10.7 & -48.49 & -43.739 & -47.229 & -1.337 & -15.1 \\
\hline $\begin{array}{c}\text { SVCDSI } \\
\text { SE }\end{array}$ & $\begin{array}{c}\text { TrK } \\
\text { B }\end{array}$ & 182 & 8.7 & -3.585 & -45.937 & -42.758 & -5.847 & -5.1 \\
\hline
\end{tabular}

\section{CONCLUSION}

In our present study, protein-peptide docking studies were performed to know the binding orientations of the BDNF peptides and TrkB receptor. These docking studies also provide in depth understanding of the interaction at their binding sites of peptide groups and receptor sites. The interacting amino acids were THR 306, ASN 6, LYS 308, LYS 4, CYS 5 and ASP 370. This protein-peptide docking studies of BDNF and TrkB provides a great assistance in understanding structural details and may lead to the establishments of therapeutic approaches in designing of effective drugs for diabetic mellitus.

\section{ACKNOWLEDGMENTS}

This Research was solely funded by Department of Science and Technology, Government of India under IRHPA Scheme vide $\mathrm{Lr}$ No. IR/SO/LU/03/2008.

\section{REFERENCES}

[1] Cochran, A. G. (2000) Chem. Biol. 7, R85-R94..

[2] Toogood, P. L. (2002) J. Med. Chem. 45, 1543-1558.

[3] Berg, T. (2003) Angew. Chem. Int. Ed. 42, 2462-2481.

[4] Eisenberg D, Marcotte E M, Xenarios I and Yeates T O 2000 Protein function in the post-genomic era; Nature (London) 405 823-826.

[5] Cusick ME, Klitgord N, Vidal M, Hill DE. Interactome: gateway into systems biology. Hum.Mol. Genet. 14(2), R171-R181 (2005).

[6] Vidal M. A unifying view of 21st century systems biology. FEBS Lett. 583(24), 3891-3894 (2009).

[7] White AW, Westwell AD, Brahemi G (2008) Proteinprotein interactions as targets for small-molecule therapeutics in cancer. Expert Reviews in Molecular Medicine 10: 1-14.

[8] Fry DC, Vassilev LT (2005) Targeting protein-protein interactions for cancer therapy. Journal of Molecular Medicine 83: 955-963.

[9] Arkin M (2005) Protein-protein interactions and cancer: small molecules going in for the kill. Current Opinion in Chemical Biology 9: 317-324.

[10] Benyamini, Future M 1002 ed. Chem. (2010) 2(6). 
[11] Pettit, F. K.; Bowie, J. U. J. Mol. Biol., 1999, 285, 1377.

[12] Lewin GR, Barde WA. Physiology of the neurotrophins. Annu Rev Neurosci 1996;19:289 - 317.

[13] Mattson MP, Maudsley S, Martin B (2004) A neural signaling triumvirate that influences ageing and agerelated disease: Insulin/IGF-1, BDNF and serotonin. Ageing Res Rev 3: 445-464.

[14] Reichardt LF (2006) Neurotrophin-regulated signaling pathways. Philos Trans R Soc Lond B Biol Sci 361:1545-1564.

[15] Barde YA (1994) Neurotrophins: A family of proteins supporting the survival of neurons. Prog Clin Biol Res 390: 45-56.

[16] Pedersen BK, Pedersen M, Krabbe KS, et al. Role of exerciseinduced brain-derived neurotrophic factor production in the regulation of energy homeostasis in mammals. Exp Physiol 2009;94:1153e1160.
[17] Nakagawa T, Tsuchida A, Itakura Y, et al. Brain-derived neurotrophic factor regulates glucose metabolism by modulating energy balance in diabetic mice. Diabetes 2000;49:436- 44.

[18] Tsuchida A, Nonomura T, Nakagawa T, et al. Brainderived neurotrophic factor ameliorates lipid metabolism in diabetic mice. Diabetes Obes Metab 2002;4:262 - 9.

[19] Tsuchida A, Nonomura T, Kishino M, et al. Acute effects of brain derived neurotrophic factor on energy expenditure in obese diabetic mice. Int $\mathbf{J}$ Obes Relat Metab Disord 2001;25:1286- 93.

[20] Ono M, Ichihara J, Nonomura T, Itakura Y, Taiji M, Nakayama C and Noguchi H (1997) Brain-derived neurotrophic factor reduces blood glucose level in obese diabetic mice but not in normal mice. Biochem Biophys Res Commun 238, 633-637. 